\title{
Photoactivation of 9-hydroxypheophorbide $\alpha$ triggers apoptosis through the reactive oxygen species-mediated mitochondrial pathway and endoplasmic reticulum stress in AMC-HN-3 laryngeal cancer cells
}

\author{
PEIJIE HE ${ }^{1,2,3}$, JIN-CHUL AHN ${ }^{1,2}$, JANG-IN SHIN ${ }^{2}$ and PHIL-SANG CHUNG ${ }^{1,2}$ \\ ${ }^{1}$ Department of Otolaryngology - Head and Neck Surgery and ${ }^{2}$ Medical Laser Research Center, College of Medicine, \\ Dankook University, Cheonan 330-715, Republic of Korea; ${ }^{3}$ Department of Otolaryngology - Head and Neck Surgery, \\ Eye, Ear, Nose and Throat Hospital affiliated to Fudan University, Shanghai 200031, P.R. China
}

Received October 20, 2009; Accepted December 21, 2009

DOI: 10.3892/ijo_00000556

\begin{abstract}
Photodynamic therapy (PDT) is a promising treatment modality for a variety of cancers. It utilizes lightabsorbing compounds combined with direct illumination to generate reactive oxygen species in photosensitizer-targeted tumor cells resulting in the final photodamage of tumors. Recently, we demonstrated that a combination modality of 9-hydroxypheophorbide $\alpha$ (9-HPbD)-based PDT and carboplatin exerts enhanced cytotoxic and apoptotic effects on AMC-HN-3 laryngeal cancer cells. The present study aimed to investigate the potential apoptotic pathways initiated by 9-HPbD-PDT-mediated reactive oxygen species (ROS) in AMC-HN-3 cells. Cytotoxicity and apoptosis induced by 9-HPbD-PDT were exhibited in a ROS-dependent manner. Mitochondria and the endoplasmic reticulum (ER) were observed as preferential sites of 9-HPbD accumulation in AMC-HN-3 cells. ROS induced by 9-HPbD-PDT directly led to downregulated expression of $\mathrm{Bcl}-2$, loss of mitochondrial membrane potential, release of cytochrome c from mitochondria, elevation of intracellular calcium due to ER stress, as well as induction of CHOP and activation of caspase- $3,-8$, -9 and -12 . Our results demonstrated that ROS induced by 9-HPbD-PDT play a causative role in triggering mitochondrial events, ER stress and probable involvement of the extrinsic apoptotic pathway in AMC-HN-3 cells.
\end{abstract}

Correspondence to: Dr Phil-Sang Chung, Department of Otolaryngology - Head and Neck Surgery, College of Medicine, Dankook University, Cheonan 330-715, Republic of Korea E-mail: pschung@dankook.ac.kr

Key words: photodynamic therapy, 9-hydroxypheophorbide- $\alpha$, reactive oxygen species, laryngeal cancer, mitochondria, endoplasmic reticulum, intracellular calcium, apoptosis

\section{Introduction}

Production of reactive oxygen species (ROS) is one of the mechanisms shared by all non-surgical therapeutic approaches to cancers, including chemotherapy, radiotherapy and photodynamic therapy (PDT) (1). The cell death mechanism induced by ROS has become a hotspot of research in the treatment of malignant diseases (1-4).

PDT is a novel and promising anticancer modality utilizing ROS through activation of a photosensitizer following visible light irradiation, which has a wavelength matching the absorption spectrum of the administered photosensitizer. Multiple signaling cascades are concomitantly activated in cancer cells exposed to photodynamic stress, and depending on the subcellular localization of the damaging ROS, these signals are transduced into adaptive or cell death responses (5). The mechanisms of PDT-mediated cell death are not fully understood. Apoptosis has been reported as the main mode of PDT-mediated cell death, but multiple signaling pathways are likely to be involved in the death process $(5,6)$. Different apoptotic pathways have been reported to be involved in the death process induced by PDT according to the target cells involved and the photosensitizer used $(5,7)$.

In spite of the novel effect of PDT in the treatment of oncologic diseases, the limitation of laser penetration to tissue and long-lasting cutaneous photosensitivity after irradiation are unavoidable (8-11), impacting to some extent the applicability of PDT to malignant tumor therapy. With a relatively longer absorbance wavelength and shorter half-life in the body, a novel pheophorbide-based photosensitizer, 9-hydroxypheophorbide- $\alpha$ (9-HPbD), displayed an apoptosis-inducing effect and growth suppression in the MFC-7 human breast cancer cell line (12). We recently showed that 9-HPbD-PDT used in combination with carboplatin enhanced cytotoxic and apoptotic effects in AMC-HN-3 laryngeal cancer cells (13).

In the present study, we investigated the detailed apoptotic mechanisms induced by 9-HPbD-PDT-mediated oxidative stress in AMC-HN-3 cells. The results indicate that, in addition to the ROS-mediated mitochondrial apoptotic pathway, parallel pathways resulting from the oxidative stress imposed on the 
endoplasmic reticulum (ER) and the extrinsic pathway are involved in 9-HPbD-PDT-induced photocytotoxic effects.

\section{Materials and methods}

Chemicals and antibodies. For cell culture, all media supplements were supplied by Hyclone (South Logan, UT, USA). Hoechst 33342 dye, propidium iodide (PI) dye, RIPA buffer, L-glutathione (GSH) and L-ascorbic acid were purchased from Sigma-Aldrich (St. Louis, MO, USA). CE1 and CE2 extraction buffer were purchased from Qiagen (Valencia, CA, USA). Caspase-3 was purchased from Calbiochem (San Diego, CA, USA), Bcl-2 and caspase-9 were from Santa Cruz Biotechnology (Santa Cruz, CA, USA), GAPDH was purchased from Abcam (Cambridge, UK), caspase-12 was from Chemicon (Temecula, CA, USA), $\mathrm{C} / \mathrm{EBP}$ homologous protein (CHOP) was from Affinity Bioreagents (Golden, CO, USA), and cytochrome c was from BD Pharmingen (San Jose, CA, USA). Rhodamine 123, 2',7'-dichlorodihydrofluorescein diacetate ( $\left.\mathrm{H}_{2} \mathrm{DCFDA}\right)$, MitoTracker ${ }^{\circledR}$ Green FM, ER-Tracker ${ }^{\mathrm{TM}}$ Blue-White DPX, and Calcium Green ${ }^{\mathrm{TM}}-1$ were supplied by Molecular Probes (Eugene, OR, USA).

Cell culture. The AMC-HN-3 cell line (kindly donated by Asan Medical Center, Seoul, Korea), developed from a 63-year-old male patient with previously untreated laryngeal squamous cell carcinoma (14), was cultured in RPMI-1640 medium supplemented with $10 \%$ FBS, penicillin (50 units/ $\mathrm{ml})$ and streptomycin $(50 \mu \mathrm{g} / \mathrm{ml})$ in an atmosphere of $5 \%$ $\mathrm{CO}_{2}$ and $95 \%$ air at $37^{\circ} \mathrm{C}$ in a humidified incubator.

Photodynamic therapy protocol. The novel chlorophyllderived photosensitizer, 9-HPbD (12), with a maximum absorption peak at $664 \mathrm{~nm}$ was kindly provided by Kumho Life and Environmental Science Laboratory (Kwangju, Korea). Stock solutions $(1.5 \mathrm{mg} / \mathrm{ml})$ were prepared in ethanol and stored in aluminum foil at $-20^{\circ} \mathrm{C}$. The cultured cells were incubated with various concentrations of 9-HPbD for $6 \mathrm{~h}$ at $37^{\circ} \mathrm{C}$ in the dark. Subsequently, the culture medium was changed, and the photosensitized cells were exposed to a 670 -nm diode laser at an energy density of $2.0 \mathrm{~J} / \mathrm{cm}^{2}$ for $15 \mathrm{~min}$ to activate $9-\mathrm{HPbD}$.

Antioxidant treatment. AMC-HN-3 cells were co-incubated with $0.59 \mu \mathrm{g} / \mathrm{ml} 9-\mathrm{HPbD}$ and either $5 \mathrm{mM} \mathrm{GSH}$ or $2.5 \mathrm{mM}$ ascorbic acid for $6 \mathrm{~h}$ in a dark incubator. After being washed with fresh medium, the cells were subjected to irradiation under the aforementioned conditions.

ROS measurement. Briefly, $1 \mathrm{~h}$ after PDT, cells were incubated with $2 \mu \mathrm{M} \mathrm{H}_{2}$ DCFDA for 30 min and gently washed twice with DPBS. Images of green $\mathrm{H}_{2}$ DCFDA were collected with LSM-510-META confocal microscopy (Zeiss, Oberkochen, Germany) with an excitation wavelength of $488 \mathrm{~nm}$, a 560-nm dichroic mirror, and a 505- to 550-nm band pass barrier filter.

Cell viability assay. Cell viability was measured by MTT assay. Following PDT, $50 \mu \mathrm{l}$ of the MTT solution $(2 \mathrm{mg} / \mathrm{ml})$ was added to each well and incubated for $2 \mathrm{~h}$. The MTT solution was then replaced by $100 \mu \mathrm{l}$ of DMSO. After $20 \mathrm{sec}$ of shaking, the absorbance at $540 \mathrm{~nm}$ was measured, and the percentage of cell viability was calculated according to the equation: cell viability $(\%)=$ mean absorbance in the treatment group/mean absorbance in the control group x 100.

Hoechst and PI double staining. Twelve hours after PDT, the cells were stained with Hoechst 33342/PI and observed using confocal microscopy as previously described (13). 9-HPbDPDT-induced apoptosis was quantified according to the percentage of Hoechst 33342- and/or PI-positive stained cells suggestive of apoptosis with shrunken, condensed or fragmented nuclei.

Subcellular localization of $9-H P b D$. During the last $30 \mathrm{~min}$ of 9-HPbD incubation, cells were co-loaded with ER-Tracker and MitoTracker at a final concentration of $500 \mathrm{nM}$ to respectively label the ER and mitochondria. An overlapped signal from organelle probes and $9-\mathrm{HPbD}$ was observed with confocal microscopy. Organelle probes were excited with a 488-nm argon laser (MitoTracker) or a 405-nm diode laser (ER-Tracker). 9-HPbD was excited with a helium/neon laser at $633 \mathrm{~nm}$. Band-pass emission filters of 530-600 and 420-480 nm were used to discriminate organelle probes (channel 1, green) from 9-HPbD (channel 2, red) fluorescence, respectively. A long pass emission filter of $650 \mathrm{~nm}$ was used for 9-HPbD.

Assessment of mitochondrial membrane potential (MMP). In brief, 9-HPbD-PDT-treated cells were loaded with $1 \mu \mathrm{M}$ rhodamine 123 for $30 \mathrm{~min}$. After gentle washing, cells were observed with confocal microscopy using the same filter settings as used for $\mathrm{H}_{2}$ DCFDA. MMP was further monitored by flow cytometry (Becton Dickinson, San Jose, CA, USA). The suspended cells were loaded with $1 \mu \mathrm{M}$ rhodamine 123 for $30 \mathrm{~min}$ and detected using the FL1-H channel. At least 20,000 events were counted.

Detection of intracellular calcium. EGTA ( $2 \mathrm{mM})$ was added to chelate any residual $\mathrm{Ca}^{2+}$ in the RPMI-1640 medium (15). The photosensitized cells were incubated with $\mathrm{Ca}^{2+}$-free medium and irradiated, $2 \mathrm{~h}$ following which cells were incubated with $4 \mu \mathrm{M}$ Calcium Green-1 for $30 \mathrm{~min}$ and observed by confocal microscopy using the same filter settings as for $\mathrm{H}_{2} \mathrm{DCFDA}$. Intracellular $\mathrm{Ca}^{2+}$ was also monitored by flow cytometry following the same procedures described for rhodamine 123 .

Western blot analysis. Using CE1/CE2 and RIPA buffers, the cytosolic/membranous and total proteins were respectively extracted 3 and $9 \mathrm{~h}$ after PDT. Equivalent amounts of protein $(100 \mu \mathrm{g})$ were loaded onto $10 \%$ polyacrylamide gels, subjected to electrophoresis, and transferred to a polyvinylidene fluoride membrane. After blocking with 5\% skim milk for $1 \mathrm{~h}$, primary antibodies were bound overnight at $4^{\circ} \mathrm{C}$. Each membrane was probed with horseradish peroxidaseconjugated anti-mouse or anti-rabbit IgG antibody for $1 \mathrm{~h}$. Labeled protein bands were detected by a Kodak in vivo image analyzer (Eastern Kodak, Rochester, NY, USA). 


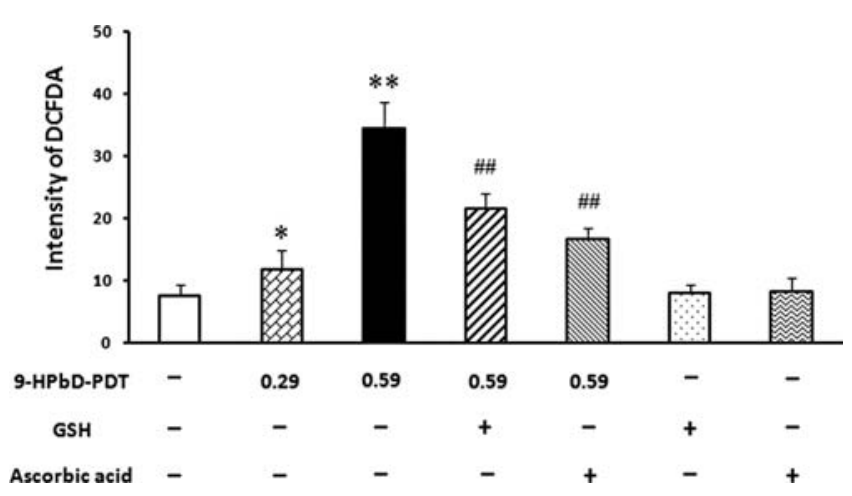

Figure 1. Accumulation of ROS after photoactivation of 9-HPbD. ROS were measured $1 \mathrm{~h}$ after irradiation. Intensity of the fluorescence signal was calculated using Image $J$ in the public domain (service at http://rsb. info.nih.gov/ij/). Bars represent the average $( \pm$ SD) of three separate experiments. ${ }^{*} \mathrm{p}<0.05,{ }^{* *} \mathrm{p}<0.01$ vs control; ${ }^{\# \#} \mathrm{p}<0.01$ vs PDT.

Statistical analysis. Results are expressed as the mean \pm standard deviation (SD). The significance of differences was evaluated by one-way analysis of variance (ANOVA) followed by the Least Significant Difference Procedure (LSD). Differences were considered statistically significant at $\mathrm{p}<0.05$.

\section{Results}

PDT-dependent ROS formation. Intracellular ROS formation was monitored by the conversion of non-fluorescent $\mathrm{H}_{2}$ DCFDA to fluorescent 2,7-dichlorofluorescein diacetate (DCFDA). As shown in Fig. 1, ROS initiated by 9-HPbDPDT exhibited a photosensitizer dose-dependent manner, which was significantly inhibited by a 6-h pretreatment with GSH or ascorbic acid $(\mathrm{p}<0.01$, respectively). The production of general ROS dramatically increased within $30 \mathrm{~min}$, then peaked at $1 \mathrm{~h}$ after 9 -HPbD-PDT. Thereafter, the intensity was attenuated gradually from 1-6 h following PDT (data not shown).

Photocytotoxic effect of 9-HPbD. In the presence of light illumination, the photocytotoxicity of $9-\mathrm{HPbD}$ exhibited a time- and photosensitizer dose-dependent response (Fig. 2A). The photodamage induced by 9-HPbD-PDT was significantly inhibited by pretreatment with GSH or ascorbic acid $(\mathrm{p}<0.01$, respectively) (Fig. 2B).

Induction of apoptosis by 9-HPbD-PDT. The apoptotic cell death, initiated in a dose-dependent manner by $9-\mathrm{HPbD}$ PDT, was significantly inhibited by pretreatment with GSH or ascorbic acid (Fig. 3; p $<0.01$, respectively). At higher concentrations of 9-HPbD-PDT (1.17-4.69 $\mu \mathrm{g} / \mathrm{ml})$, a gradually increasing proportion of necrotic cells was evident (data not shown). A time-dependent apoptotic effect was also observed 3-24 h after PDT (data not shown).

Localization of 9-HPbD to the mitochondria and ER. Subcellular localization of the photosensitizer is usually at the site of photodamage (5). As illustrated in Fig. 4, the fluorescent signal of $9-\mathrm{HPbD}$ overlapped with that from the mitochondria and ER in merged images, suggesting that
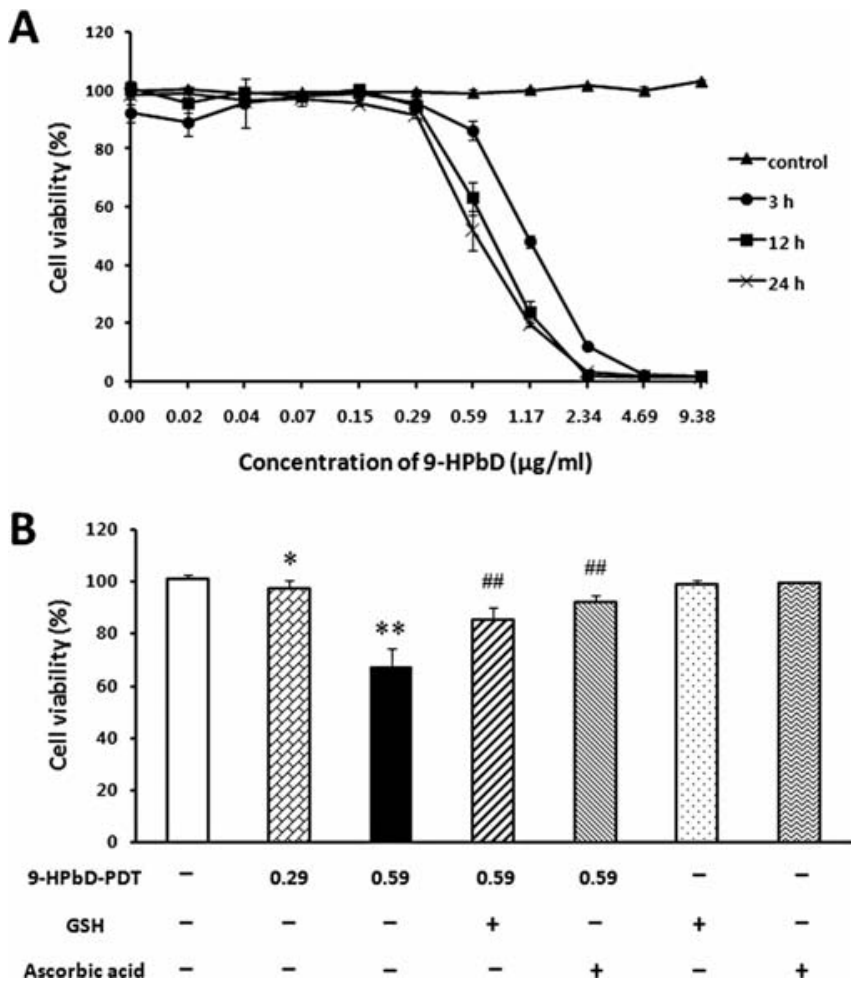

Figure 2. Cytotoxicity of 9-HPbD-mediated PDT on AMC-HN-3 cells. (A) Cell viability was assessed 3,12 and $24 \mathrm{~h}$ after PDT. (B) Protective effect of antioxidants on 9-HPbD-mediated photodynamic damage. MTT assay was performed $12 \mathrm{~h}$ after PDT. Data shown represent the average $( \pm \mathrm{SD})$ of three independent experiments. ${ }^{*} \mathrm{p}<0.05,{ }^{* *} \mathrm{p}<0.01$ vs control; ${ }^{\# \#} \mathrm{p}<0.01$ vs PDT.

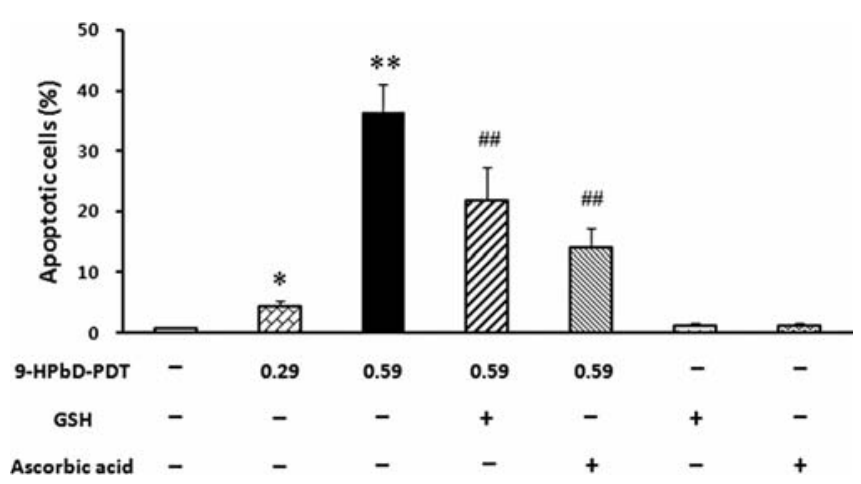

Figure 3. Apoptosis induced by 9-HPbD-PDT in AMC-HN-3 cells. Hoechst 33342 and PI staining was performed $12 \mathrm{~h}$ after PDT. The percentage of apoptotic cells was calculated according to the numbers of Hoechst 33342 and/or PI-positive-stained cells. Bars represent the average $( \pm$ SD) of three separate experiments. ${ }^{*} \mathrm{p}<0.05,{ }^{* *} \mathrm{p}<0.01$ vs control; ${ }^{\# \#} \mathrm{p}<0.01$ vs PDT.

these AMC-HN-3 cell organelles were the preferential sites of 9-HPbD accumulation after a 6-h incubation.

9-HPbD-PDT initiates mitochondrial depolarization. The polarized mitochondria were imaged as bright fluorescent spheres and tubes. After 9-HPbD-PDT exposure, the majority of the bright spheres disappeared, and the signal intensity of rhodamine 123 became faint and diffused, indicating mitochondrial depolarization (Fig. 5A). As shown in Fig. 5B and $\mathrm{C}$, a leftward shift of the fluorescence curve, indicative of collapsed MMP, was observed in a 9-HPbD dose-dependent 


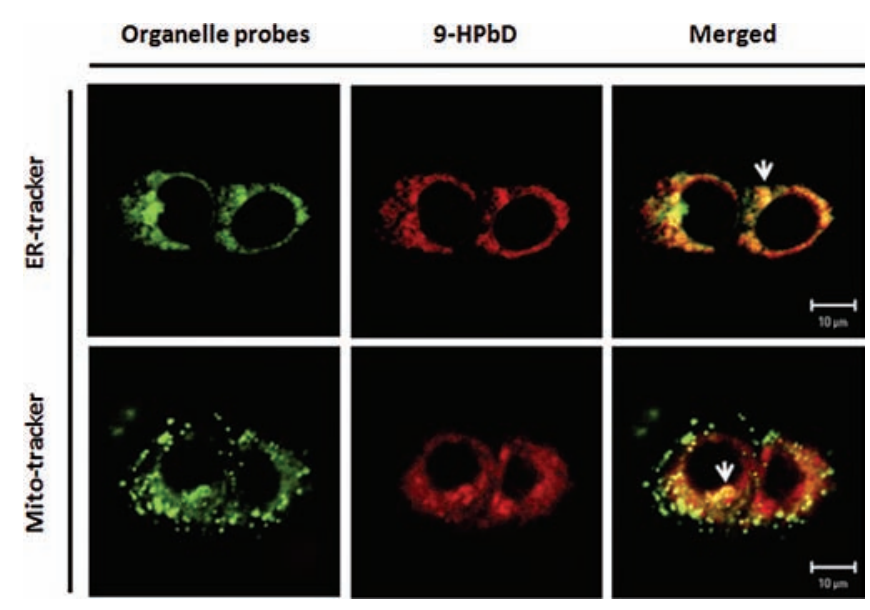

Figure 4. Subcellular localization of 9-HPbD. AMC-HN-3 cells were coloaded with $9-\mathrm{HPbD}$ and organelle probes and were detected by confocal microscopy. Arrows in the merged images point to the co-localized area of fluorescence signals from 9-HPbD and ER-/MitoTracker. Scale bar, $10 \mu \mathrm{m}$.

manner. Meanwhile, a time-dependent pattern was also exhibited; the cells with disruption of MMP gradually increased 1-6 h following PDT (data not shown). PDTinduced collapse of MMP was clearly inhibited by pretreatment with GSH or ascorbic acid 2 and $6 \mathrm{~h}$ after irradiation, indicating the causative roles of ROS in mitochondrial depolarization (Fig. 5D-G).

9-HPbD-PDT leads to elevation of intracellular $\mathrm{Ca}^{2+}$. Due to the use of $\mathrm{Ca}^{2+}$-free medium, $\mathrm{Ca}^{2+}$ influx from the medium was blocked. Thus, alteration in the intracellular $\mathrm{Ca}^{2+}$ level reflected the perturbation of $\mathrm{Ca}^{2+}$ stores in the ER and mitochondria. As shown in Fig. 6A-C, 9-HPbD-PDT induced a significant $\mathrm{Ca}^{2+}$ elevation $2 \mathrm{~h}$ post-irradiation $(\mathrm{p}<0.01)$, exhibiting a dose-dependent response (Fig. 6C). The increase in the intracellular $\mathrm{Ca}^{2+}$ level was significantly inhibited by pretreatment with GSH or ascorbic acid (Fig. 6A, B, D and E). There was no obvious impact on the intracellular $\mathrm{Ca}^{2+}$ level while the cells were treated with GSH or ascorbic acid alone (Fig. 6A and B).

Apoptosis signaling pathways induced by 9-HPbD-PDT. The release of cytochrome $\mathrm{c}$ from the mitochondria to the cytosol was suggestive of mitochondrial activation. As shown in Fig. 7A, the amount of cytosolic cytochrome $\mathrm{c}$ was significantly increased $3 \mathrm{~h}$ after PDT, and this increase was partly inhibited by pretreatment with antioxidants. Compared with the control group, there was no noticeable expression of cytosolic cytochrome $\mathrm{c}$ in the antioxidant-pretreatment samples. In contrast, the level of Bcl-2 in the membranous fraction significantly decreased $3 \mathrm{~h}$ after PDT, and this downregulation of $\mathrm{Bcl}-2$ was also partly inhibited by pretreatment with GSH or ascorbic acid (Fig. 7B).

Since 9-HPbD localized, not only in the mitochondria but also in the ER, it was logical that the photoactivation of 9-HPbD also induced ER stress in 9-HPbD-PDT-treated AMC-HN-3 cells. Therefore, we determined whether the ER stress-related proteins, caspase- 12 and CHOP, were activated after PDT. The expression of caspase-12 and CHOP was up-
A

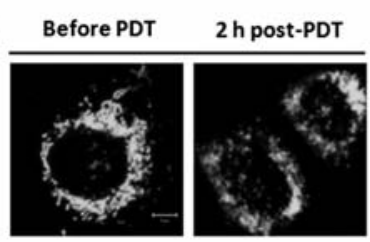

$\mathbf{B}_{8}$
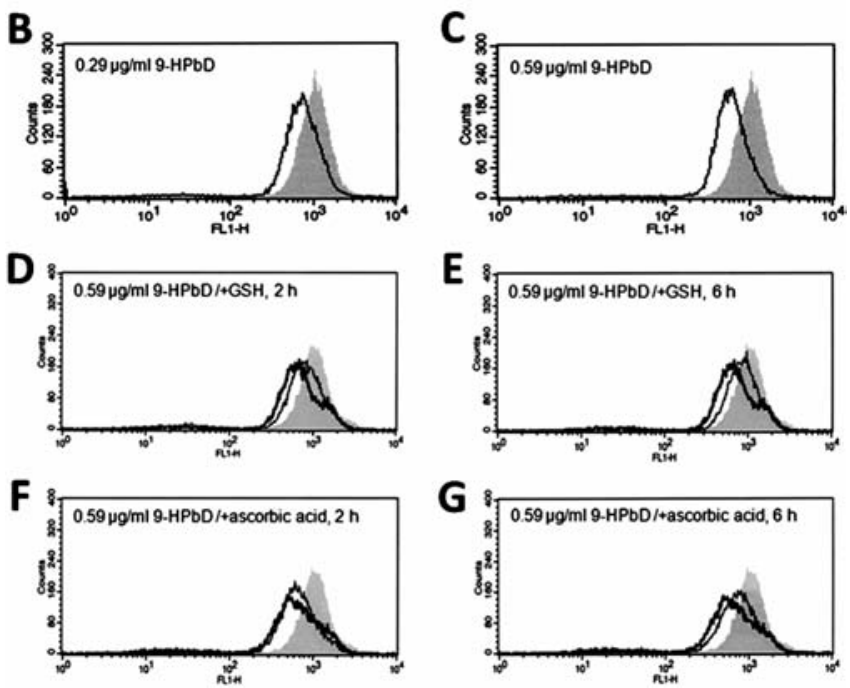

Figure 5. Protective roles of antioxidants in 9-HPbD-PDT-induced MMP collapse. MMP was detected $2 \mathrm{~h}$ following $0.59 \mu \mathrm{g} / \mathrm{ml}$ 9-HPbD-PDT by confocal microscopy (A) and flow cytometry (B and C depict 0.29 and $0.59 \mu \mathrm{g} / \mathrm{ml}$ 9-HPbD-PDT, respectively). The distribution of the control (non-treated) group is shown as a gray-shaded area, and the PDT groups as a solid contour (B and C). MMP was monitored 2 and $6 \mathrm{~h}$ after $0.59 \mu \mathrm{g} / \mathrm{ml}$ 9-HPbD-PDT with or without pretreatment of GSH (D and E) or ascorbic acid (F and G). The PDT groups are depicted as a bold contour, and antioxidant-pretreated-PDT groups are displayed as a fine contour. The spectral shift of the fluorescence curve to the left indicates mitochondrial membrane depolarization. A typical experiment of three experiments is presented. Scale bar in A, $5 \mu \mathrm{m}$.

regulated $9 \mathrm{~h}$ after PDT (Fig. 7C), which indicated that 9-HPbD-PDT exerted ER stress on the cells. Notably, the increased cleavage of caspase-12 was down-regulated after pretreatment with the antioxidants. However, the up-regulation of CHOP was not inhibited by pretreatment with GSH but was partially suppressed by ascorbic acid (Fig. 7C).

Expression of the downstream caspase cascade, including caspase-3, -8 and -9, was also detected. As shown in Fig. 7C, 9-HPbD-PDT induced significant activation of these caspases, which were markedly inhibited by pretreatment with the antioxidants.

\section{Discussion}

The basic principle of PDT is that a photosensitizer generates ROS during activation, and the generated ROS initiates cell death (16). Using the fluorescent probe, $\mathrm{H}_{2}$ DCFDA, it was shown in the present study that 9-HPbD-PDT induced the generation of ROS. The ROS levels in 9-HPbD-treated cells, monitored $1 \mathrm{~h}$ following irradiation, were increased in a photosensitizer dose-dependent manner (Fig. 1).

GSH (17), a direct scavenger of singlet oxygen and hydroxyl radical, and ascorbic acid (18), a well-known general antioxidant which scavenges the hydroxyl radical, 
A

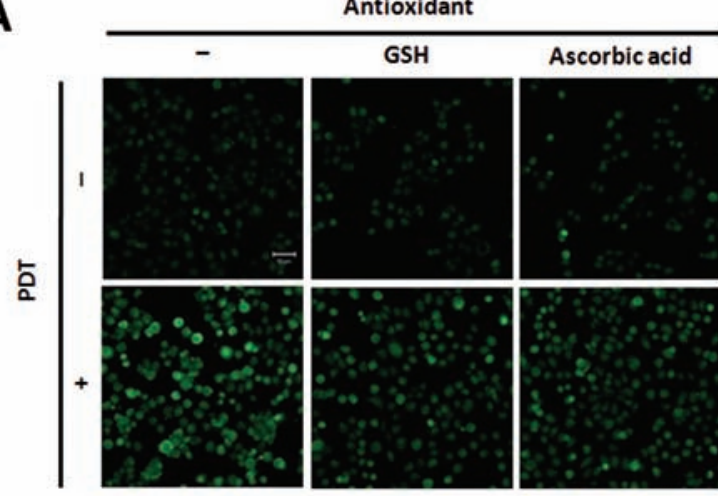

B

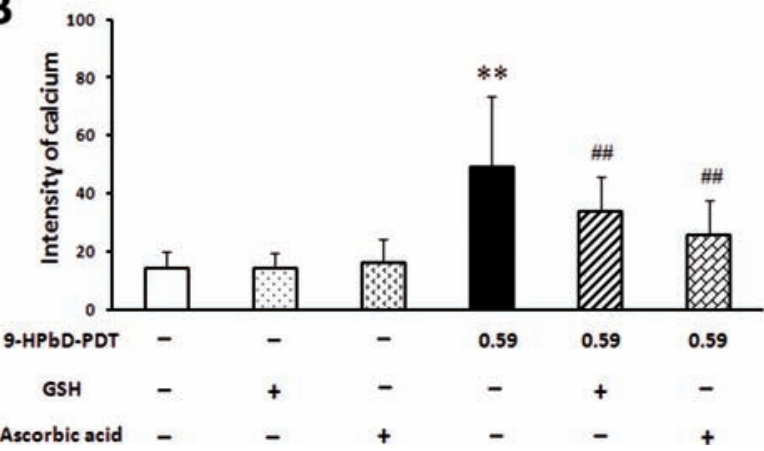

C

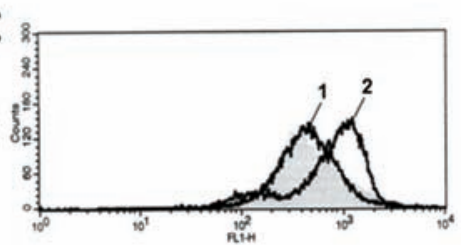

D

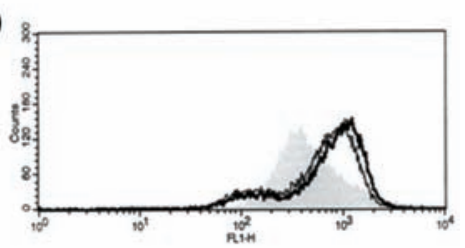

E

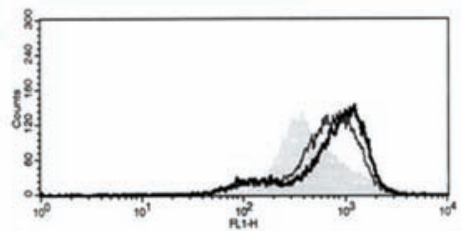

Figure 6. Intracellular $\mathrm{Ca}^{2+}$ level in response to 9-HPbD-PDT. Intracellular $\mathrm{Ca}^{2+}$ was monitored using confocal microscopy (A and B) and flow cytometry (C-E) $2 \mathrm{~h}$ after PDT. Intensity of Calcium Green-1 fluorescence in (A) was calculated through ImageJ (B). Intracellular Ca ${ }^{2+}$ induced by 0.29 and $0.59 \mu \mathrm{g} / \mathrm{ml}$ 9-HPbD-PDT is respectively illustrated in line 1 and 2 (C). Elevation of $\mathrm{Ca}^{2+}$ was partially inhibited by GSH and ascorbic acid (D and E, respectively). The distribution of the control (non-treated) group is shown as a gray-shaded area, PDT groups as a bold contour, and the antioxidant-pretreated PDT groups as a fine contour. The spectral shift of the fluorescence curve to the right indicates increased concentration of $\mathrm{Ca}^{2+}(\mathrm{C}-\mathrm{E})$. A typical experiment of three experiments is presented. Scale bar in A, $50 \mu \mathrm{m}$. Bars in B represent the average $( \pm \mathrm{SD})$ of three separate experiments. ${ }^{* *} \mathrm{p}<0.01 \mathrm{vs}$ control; ${ }^{\# \#} \mathrm{p}<0.01 \mathrm{vs}$ PDT.

A

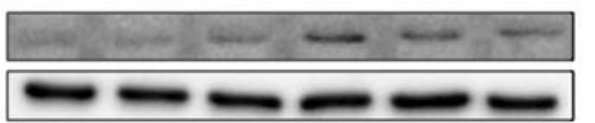

Cytochrome c

B

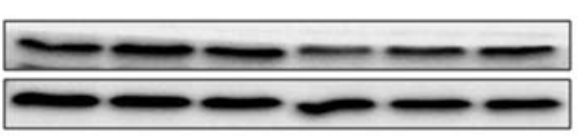

C

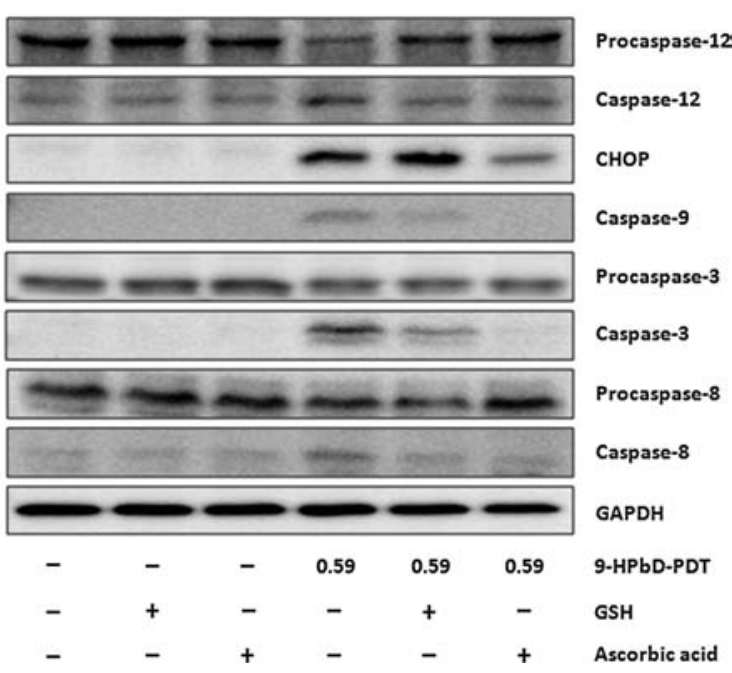

Figure 7. Expression of cytochrome c (A), Bcl-2 (B), CHOP, caspase-12, -9, -8 and -3 (C) under oxidative stress induced by $0.59 \mu \mathrm{g} / \mathrm{ml}$ 9-HPbD-PDT. Cytosolic (A), membranous (B) and total (C) proteins were respectively prepared and detected with Western blotting. GAPDH was included as a loading control. superoxide anion/hydroperoxyl radical, hydrogen peroxide, as well as singlet oxygen, were respectively used to explore the role of ROS in 9-HPbD-PDT-induced phototoxicity and apoptosis in AMC-HN-3 cells. As shown in Fig. 1, ROS generated by $9-\mathrm{HPbD}-\mathrm{PDT}$ were significantly inhibited by pretreatment with GSH or ascorbic acid.

As a new photosensitizer, 9-HPbD-PDT was found to inhibit the growth of the MCF-7 human breast cancer cell line and had an apoptosis-inducing effect (12). In the present study, the photocytotoxicity of 9-HPbD was tested in a doseand time-dependent response in AMC-HN-3 human laryngeal cancer cells. Cell viability was significantly protected by pretreatment with GSH or ascorbic acid, indicating the causative role of ROS in $9-\mathrm{HPbD}$-induced phototoxicity (Fig. 2). The apoptotic effect of 9-HPbD-PDT was also exhibited in a $9-\mathrm{HPbD}$ dose-dependent manner, which was significantly suppressed by pretreatment with GSH or ascorbic acid, suggesting that the generation of ROS plays a pivotal role in 9-HPbD-PDT-induced apoptosis (Fig. 3).

It is generally accepted that the intracellular localization of the photosensitizer coincides with the primary site of photodamage (5) since photogenerated singlet oxygen has a very short life and very limited diffusion in biological systems (half-life, $<0.04 \mu \mathrm{sec}$; radius of action, $<0.02 \mu \mathrm{m}$ ), indicating that primary molecular targets of the photodynamic process must reside within a few nanometers from the dye (16). Meanwhile, the molecular nature of the photo-oxidized targets has a profound influence on the signaling pathways and mode of cell death following PDT $(5,19)$. Therefore, the 
subcellular localization of 9-HPbD was detected to explore the potential photodamage targets and the probable signaling pathways involved. As shown in Fig. 4, 9-HPbD localized prominently in the ER and mitochondria of AMC-HN-3 cells, which prompted us to further examine the mitochondrial apoptotic events and ER stress-associated apoptosis.

Mitochondria are increasingly recognized as an important target organelle during the photodamage of tumor cells $(5,20,21)$. In general, cell killing is preceded by a collapse of MMP, which has been implicated as a key factor which initiates a cascade of events (e.g., release of cytochrome c and the activation of caspases) leading to cell death (20-23). As illustrated in Fig. 5A, polarized mitochondria in untreated cells were imaged as bright fluorescent spheres and tubes, while $2 \mathrm{~h}$ after PDT, depolarized MMP was detected as faint and diffuse fluorescence with loss of characteristic figures. Consistent with this morphologic change, MMP collapse occured in a photosensitizer dose-dependent manner $2 \mathrm{~h}$ after PDT (Fig. 5B and C). Upon pretreatment with the antioxidants, MMP collapse was partially protected either $2 \mathrm{~h}$ (Fig. 5D and F) or $6 \mathrm{~h}$ (Fig. 5E and G) after PDT, indicating the decisive role of ROS in mitochondrial activation.

Disruption of the MMP is an early requirement for apoptosis (24) and is generally believed to be responsible for the release of several apoptogenic proteins stored in the inner-mitochondrial membrane space $(5,24,25)$. As shown in Fig. 7, the increased release of cytochrome $\mathrm{c}$ from the mitochondria to the cytosol was observed $3 \mathrm{~h}$ after PDT, which was partially suppressed by pretreatment with the antioxidants, further suggesting the causative role of ROS in mitochondrial activation. Through the formation of apoptosome, cytochrome c recruits and activates caspase-9 $(5,22)$. Activated caspase-9, as well as downstream executioner caspase-3, were up-regulated $9 \mathrm{~h}$ after PDT and this upregulation was efficiently inhibited by pretreatment with the antioxidants (Fig. 7C). Up-regulation of caspase-3 and -9 was also observed after a combined modality with 9-HPbDPDT and carboplatin in AMC-HN-3 cells (13). However, activation of caspase-9 was not detected in 9-HPbD-induced MCF-7 cell death (12). A probable different localization of 9-HPbD in MCF-7 and AMC-HN-3 cells may lead to the distinct activation of caspase-9.

The functioning of the mitochondrial membrane is also affected by members of the Bcl-2 family. Bcl-2, an antiapoptotic protein of the Bcl-2 family, promotes cell survival by interfering with the activation of the cytochrome c/Apaf-1 pathway through stabilization of the mitochondrial membrane (26) and is a target of some photosensitizers (27-30). In the present study, Bcl-2 was down-regulated by $9-\mathrm{HPbD}-\mathrm{PDT}$, which was prevented by pretreatment with the antioxidants (Fig. 7B), indicating the causative role of ROS in decreased expression of Bcl-2. This PDT-induced photodamage of Bcl-2 may be associated with the regulation of phosphorylation and ubiquitination under oxidative stress.

The indicators suggestive of mitochondrial events, including the decrease in the organelle membranous level of Bcl-2 (Fig. 7B), the disruption of MMP (Fig. 5), enhanced release of cytochrome $\mathrm{c}$ to the cytosol, activation of caspase-9 and -3 (Fig. 7A and C), along with the efficient suppression of these mitochondrial events by the two types of anti-

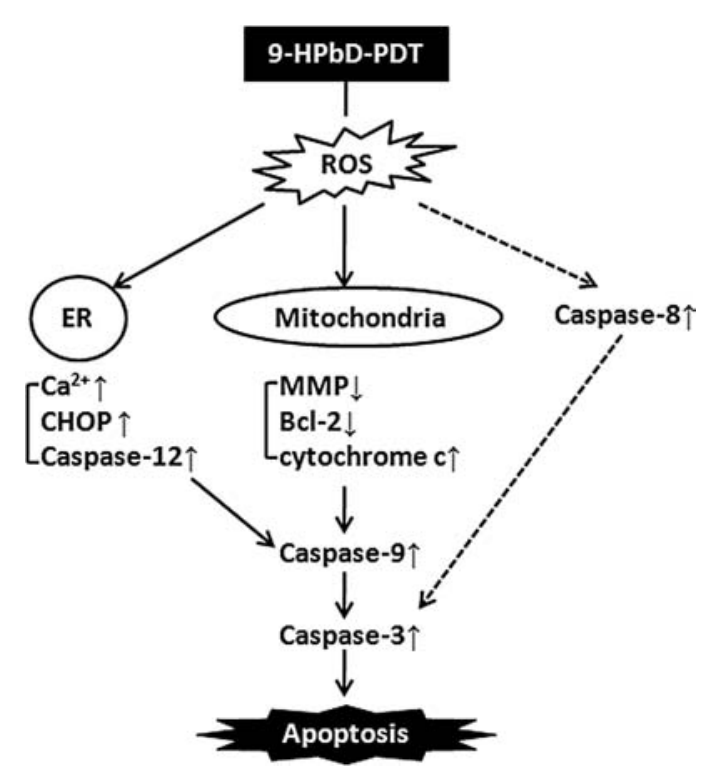

Figure 8. Apoptosis pathways triggered by $9-\mathrm{HPbD}-\mathrm{PDT}$-induced oxidative stress.

oxidants, demonstrate the causative role of ROS in mitochondrial photodamage.

Generally, PDT exerts oxidative stress on cells. Through this, perturbation of the ER function may occur causing cell photodamage in the case of using an ER-localized photosensitizer $(27,29)$. Since 9-HPbD localized, not only in the mitochondria but also in the ER of the AMC-HN-3 cells (Fig. 4), an ER photodamage-related signal pathway is logically involved in 9-HPbD PDT-induced apoptosis.

ER stress-induced apoptotic cell death represents a novel mitochondrial-independent intrinsic apoptotic pathway (31). Caspase-12-deficient mouse embryonic fibroblast cells were found to exhibit partial resistance, specifically against ER stress-inducing agents, suggesting an important role for this caspase (32). In the present study, the activation of caspase-12 was observed in AMC-HN-3 cells after PDT, and this upregulation was attenuated by pretreatment with antioxidants (Fig. 7C), indicating the important role of ROS in the induction of ER stress. Caspase-9 is documented as the downstream substrate of caspase-12 independent of cytochrome c (33). As shown in Fig. 7C, activation of caspase-12 and -9 shared the same response pattern to oxidative stress induced by PDT, suggesting the initiation of the ER stressspecific caspase cascade in apoptosis.

CHOP is an ER stress inducible transcription factor involved in the development of apoptosis, growth arrest, and differentiation. CHOP induction is thought to tip the balance towards apoptosis under conditions of persistent ER stress (34). The enhanced apoptosis and toxicity in chop $^{+/+}$cells following PDT compared to identical treatments in chop $^{-1}$ cells, suggests an important role of CHOP in PDT-induced apoptosis (35). Induction of CHOP was clearly observed in AMC-HN-3 cells $9 \mathrm{~h}$ following PDT (Fig. 7C). In contrast to the response of caspase- 12 to the antioxidants, CHOP induction was only suppressed by pretreatment with ascorbic acid, but not by GSH. GSH did not suppress but rather enhanced the induction of CHOP by PDT (Fig. 7C), which 
implies the possibility that other types of ROS may play more important roles than singlet oxygen and the hydroxyl radical in the induction of $\mathrm{CHOP}$, even though direct and indirect evidence supports a prevalent role for singlet oxygen in the molecular processes initiated by PDT (36).

Besides the main location on the outer mitochondrial membrane, Bcl-2 also locates in the ER and the nuclear membrane (26). CHOP has also been reported to be involved in ER stress-induced apoptosis by reducing the expression of Bcl-2 (37). Similar to the induction of CHOP following PDT (Fig. 7C), the loss of the organelle membranous level of Bcl-2 (Fig. 7B) likely reflects the activation of ER induced by 9-HPbD-PDT.

ER stress also leads to increased cytosolic $\mathrm{Ca}^{2+}$, which induces the activation of m-calpain. This activated m-calpain subsequently cleaves Bcl-XL and proteolytically activates caspase-12 (38). The increase in cytosolic $\mathrm{Ca}^{2+}$ was observed in AMC-HN-3 cells $2 \mathrm{~h}$ after PDT in a photosensitizer dosedependent manner (Fig. 6C), which was partly prevented by pretreatment with the antioxidants (Fig. 6A, B, D and E). $\mathrm{Ca}^{2+}$-free medium was used before PDT, which indicated that the PDT-induced elevation in cytosolic $\mathrm{Ca}^{2+}$ was due to the emptying of $\mathrm{Ca}^{2+}$ from the ER and possibly from mitochondrial stores, and not due to an influx from the medium. The increase in $\mathrm{Ca}^{2+}$ release from the ER (Fig. 6A-C), along with the activation of caspase-12 and induction of $\mathrm{CHOP}$ (Fig. 7C), and their responses to the antioxidants manifest the induction of ER stress due to the oxidative status initiated by 9-HPbD-PDT.

In addition to the involvement of the intrinsic mitochondria- $(20,21)$ and ER-mediated $(27,29)$ apoptotic pathways described, the death receptor-mediated extrinsic apoptotic pathway has also been reported in PDT-induced cell death $(39,40)$. Although the recruitment of Fas, TNF- $\alpha$ or related death receptors was not detected herein, as an adaptor molecule of death-inducing signaling complexes, procaspase- 8 cleavage into its active form was observed concomitant with the activation of the other caspases (Fig. 7C), which was partly hindered by pretreatment with the antioxidants, implying the likely contribution of the extrinsic apoptotic pathway to 9-HPbD-PDT-mediated apoptosis under oxidative stress.

In conclusion, 9-HPbD-mediated PDT triggers apoptosis of AMC-HN-3 human laryngeal cancer cells by co-activation of the mitochondria and ER, as well as the probable parallel involvement of the extrinsic signaling pathway (Fig. 8). This is supported by the main localization of $9-\mathrm{HPbD}$ in the ER and mitochondria of AMC-HN-3 cells, initiation of mitochondrial events, induction of ER stress, and converging activation of caspase cascades including caspase-12, -9, -8 and -3 . Investigation of the molecular mechanisms also revealed that ROS act as an upstream mediator for the 9-HPbD-PDTinduced apoptosis pathways. The present findings may have useful implications for the potential application of 9-HPbDPDT in anti-neoplastic therapy. Here, we provide additional knowledge concerning its molecular action in cancer cells. Understanding the precise pathways involving the induction of apoptosis may help in the development of treatment modalities of PDT in malignant diseases, either alone or in combination with other therapeutic arms $(13,41)$.

\section{Acknowledgements}

This work was funded by Dankook Medical Laser and Device Regional Innovation Center.

\section{References}

1. Wang $\mathrm{J}$ and Yi $\mathrm{J}$ : Cancer cell killing via ROS: to increase or decrease, that is the question. Cancer Biol Ther 7: 1875-1884, 2008.

2. Kamuhabwa AR, Huygens A, Roskams T and De Witte PA: Enhancing the photodynamic effect of hypericin in human bladder transitional cell carcinoma spheroids by the use of the oxygen carrier, perfluorodecalin. Int J Oncol 28: 775-780, 2006.

3. Park JH, Kim EJ, Jang HY, et al: Combination treatment with arsenic trioxide and sulindac enhances apoptotic cell death in lung cancer cells via activation of oxidative stress and mitogenactivated protein kinases. Oncol Rep 20: 379-384, 2008.

4. Portanova P, Russo T, Pellerito O, et al: The role of oxidative stress in apoptosis induced by the histone deacetylase inhibitor suberoylanilide hydroxamic acid in human colon adenocarcinoma HT-29 cells. Int J Oncol 33: 325-331, 2008.

5. Buytaert E, Dewaele M and Agostinis P: Molecular effectors of multiple cell death pathways initiated by photodynamic therapy. Biochim Biophys Acta 1776: 86-107, 2007.

6. Moan J and Berg K: Photochemotherapy of cancer: experimental research. Photochem Photobiol 55: 931-948, 1992.

7. Oleinick NL and Evans HH: The photobiology of photodynamic therapy: cellular targets and mechanisms. Radiat Res 150: S146-S156, 1998.

8. Copper MP, Tan IB, Oppelaar H, Ruevekamp MC and Stewart FA: Meta-tetra(hydroxyphenyl) chlorin photodynamic therapy in early-stage squamous cell carcinoma of the head and neck. Arch Otolaryngol Head Neck Surg 129: 709-711, 2003.

9. Biel MA: Photodynamic therapy treatment of early oral and laryngeal cancers. Photochem Photobiol 83: 1063-1068, 2007.

10. Dolmans DE, Fukumura D and Jain RK: Photodynamic therapy for cancer. Nat Rev Cancer 3: 380-387, 2003.

11. Sibata CH, Colussi VC, Oleinick NL and Kinsella TJ: Photodynamic therapy in oncology. Expert Opin Pharmacother 2: 917-927, 2001.

12. Choi SE, Sohn S, Cho JW, Shin EA, Song PS and Kang Y: 9-Hydroxypheophorbide alpha-induced apoptotic death of MCF-7 breast cancer cells is mediated by c-Jun N-terminal kinase activation. J Photochem Photobiol B 73: 101-107, 2004.

13. He P, Ahn JC, Shin JI, et al: Enhanced apoptotic effect of combined modality of 9-hydroxypheophorbide alpha-mediated photodynamic therapy and carboplatin on AMC-HN-3 human head and neck cancer cells. Oncol Rep 21: 329-334, 2009.

14. Kim SY, Chu KC, Lee HR, Lee KS and Carey TE: Establishment and characterization of nine new head and neck cancer cell lines. Acta Otolaryngol 117: 775-784, 1997.

15. Biswas D, Qureshi OS, Lee WY, Croudace JE, Mura M and Lammas DA: ATP-induced autophagy is associated with rapid killing of intracellular mycobacteria within human monocytes/ macrophages. BMC Immunol 9: 35, 2008.

16. Moan J and Berg K: The photodegradation of porphyrins in cells can be used to estimate the lifetime of singlet oxygen. Photochem Photobiol 53: 549-553, 1991.

17. Valko M, Leibfritz D, Moncol J, Cronin MT, Mazur M and Telser J: Free radicals and antioxidants in normal physiological functions and human disease. Int J Biochem Cell Biol 39: 44-84, 2007.

18. Carr A and Frei B: Does vitamin C act as a pro-oxidant under physiological conditions? FASEB J 13: 1007-1024, 1999.

19. Oleinick NL, Morris RL and Belichenko I: The role of apoptosis in response to photodynamic therapy: what, where, why, and how. Photochem Photobiol Sci 1: 1-21, 2002.

20. Tang PM, Chan JY, Au SW, et al: Pheophorbide $\alpha$, an active compound isolated from Scutellaria barbata, possesses photodynamic activities by inducing apoptosis in human hepatocellular carcinoma. Cancer Biol Ther 5: 1111-1116, 2006.

21. Lai JC, Lo PC, Ng DK, et al: BAM-SiPc, a novel agent for photodynamic therapy, induces apoptosis in human hepatocarcinoma HepG2 cells by a direct mitochondrial action. Cancer Biol Ther 5: 413-418, 2006. 
22. Li P, Nijhawan D, Budihardjo I, et al: Cytochrome c and dATPdependent formation of Apaf-1/caspase-9 complex initiates an apoptotic protease cascade. Cell 91: 479-489, 1997.

23. Lemasters JJ, Nieminen AL, Qian T, et al: The mitochondrial permeability transition in cell death: a common mechanism in necrosis, apoptosis and autophagy. Biochim Biophys Acta 1366: 177-196, 1998

24. Ly JD, Grubb DR and Lawen A: The mitochondrial membrane potential (deltapsi(m)) in apoptosis; an update. Apoptosis 8: 115-128, 2003.

25. Lam M, Oleinick NL and Nieminen AL: Photodynamic therapyinduced apoptosis in epidermoid carcinoma cells. Reactive oxygen species and mitochondrial inner membrane permeabilization. J Biol Chem 276: 47379-47386, 2001.

26. Kluck RM, Bossy-Wetzel E, Green DR and Newmeyer DD: The release of cytochrome $\mathrm{c}$ from mitochondria: a primary site for Bcl-2 regulation of apoptosis. Science 275: 1132-1136, 1997.

27. Kessel D, Castelli M and Reiners JJ: Ruthenium red-mediated suppression of $\mathrm{Bcl}-2$ loss and $\mathrm{Ca}(2+)$ release initiated by photodamage to the endoplasmic reticulum: scavenging of reactive oxygen species. Cell Death Differ 12: 502-511, 2005.

28. Ichinose S, Usuda J, Hirata T, et al: Lysosomal cathepsin initiates apoptosis, which is regulated by photodamage to $\mathrm{Bcl}-2$ at mitochondria in photodynamic therapy using a novel photosensitizer, ATX-s10 (Na). Int J Oncol 29: 349-355, 2006

29. Buytaert E, Callewaert G, Hendrickx N, et al: Role of endoplasmic reticulum depletion and multidomain proapoptotic BAX and BAK proteins in shaping cell death after hypericin-mediated photodynamic therapy. FASEB J 20: 756-758, 2006.

30. Usuda J, Hirata T, Ichinose S, et al: Tailor-made approach to photodynamic therapy in the treatment of cancer based on Bcl-2 photodamage. Int J Oncol 33: 689-696, 2008.

31. Rao RV, Castro-Obregon S, Frankowski H, et al: Coupling endoplasmic reticulum stress to the cell death program. An Apaf-1-independent intrinsic pathway. J Biol Chem 277: 21836-21842, 2002.
32. Nakagawa T, Zhu H, Morishima N, et al: Caspase-12 mediates endoplasmic-reticulum-specific apoptosis and cytotoxicity by amyloid-beta. Nature 403: 98-103, 2000.

33. Morishima N, Nakanishi K, Takenouchi H, Shibata T and Yasuhiko Y: An endoplasmic reticulum stress-specific caspase cascade in apoptosis. Cytochrome c-independent activation of caspase-9 by caspase-12. J Biol Chem 277: 34287-34294, 2002.

34. Szegezdi E, Logue SE, Gorman AM and Samali A: Mediators of endoplasmic reticulum stress-induced apoptosis. EMBO Rep 7: 880-885, 2006.

35. Wong S, Luna M, Ferrario A and Gomer CJ: CHOP activation by photodynamic therapy increases treatment induced photosensitization. Lasers Surg Med 35: 336-341, 2004.

36. Niedre M, Patterson MS and Wilson BC: Direct near-infrared luminescence detection of singlet oxygen generated by photodynamic therapy in cells in vitro and tissues in vivo. Photochem Photobiol 75: 382-391, 2002.

37. McCullough KD, Martindale JL, Klotz LO, Aw TY and Holbrook NJ: Gadd153 sensitizes cells to endoplasmic reticulum stress by down-regulating $\mathrm{Bcl} 2$ and perturbing the cellular redox state. Mol Cell Biol 21: 1249-1259, 2001.

38. Nakagawa T and Yuan J: Cross-talk between two cysteine protease families. Activation of caspase- 12 by calpain in apoptosis. J Cell Biol 150: 887-894, 2000.

39. Olivo $\mathrm{M}$ and Ali-Seyed $\mathrm{M}$ : Apoptosis signalling mechanisms in human cancer cells induced by calphostin-PDT. Int J Oncol 30: 537-548, 2007.

40. Ruhdorfer S, Sanovic R, Sander V, Krammer B and Verwanger T: Gene expression profiling of the human carcinoma cell line A-431 after 5-aminolevulinic acid-based photodynamic treatment. Int J Oncol 30: 1253-1262, 2007.

41. Yanase S, Nomura J, Matsumura Y, Nagata T, Fujii T and Tagawa T: Synergistic interaction of 5-aminolevulinic acidbased photodynamic therapy with simultaneous hyperthermia in an osteosarcoma tumor model. Int J Oncol 29: 365-373, 2006. 